(pp 301-305)

\title{
Study on the Gender Difference of Lumbar Multifidus Cross-sectional Area Using Ultrasound System
}

\author{
Hitomi UBUKATA, PT, MS \\ Department of Physical Therapy, Faculty of Health Sciences, Takasaki University of Health and Welfare
}

Hitomi UBUKATA, PT, MS, Hitoshi MARUYAMA, PT, PhD

Department of Physical Therapy, Health and Welfare Science Course, Graduate School of International University of Health and Welfare

Ming HUO, PT, PhD

Department of Physical Therapy, Faculty of Health Care Sciences, Himeji Dokkyo University

Qiuchen HUANG, PT, MS

China Rehabilitation Research Center

Purpose: In this study, we measured the multifidus muscle cross-sectional area in normal adults using an ultrasound imaging device, to clarify factors influencing the difference in this cross-sectional area between males and females and to develop method to correct for this difference.

Methods: Subjects comprised 63 normal adults (30 males and 33 females) with no anamnestic history of lumbar pain. We measured the cross-sectional area of their lumbar multifidus muscles using an ultrasound imaging device.

Results: Regarding multifidus muscle cross-sectional area measurement, data from 25 males indicated a larger difference compared with data from females, indicating significance. However, a significant difference was not observed between males and females in multifidus muscle cross-sectional area measurement when divided by height and weight. The cross-sectional area showed a significant relationship with height, weight and BMI; however, only weight was adopted in stepwise multiple regression analysis.

Conclusions: To obtain accurate multifidus muscle cross-sectional area measurement that did not relate to the body, we suggest that this should be divided by weight. In addition, it was suggested the ratio of weight to the multifidus muscle cross-sectional area can be applied as unified basis between males and females for clinical application. 